\title{
The Translaminar Microsurgical Approach to Lumbar Disc Herniation with Foraminal Stenosis: A Modification to the Traditional Surgery Technique
}

\author{
Marcos Baabor $\mathbf{A}^{1 *}$, Hernán Delso $\mathrm{P}^{2}$ and Bayron Valenzuela $\mathrm{C}^{2}$ \\ ${ }^{1}$ Director of Neurosurgery Department, Clinical Hospital of University, Chile \\ ${ }^{2}$ Resident of Neurosurgery, Clinical Hospital of University, Chile
}

*Corresponding author: Marcos Baabor A, Director of Neurosurgery Department, Clinical Hospital of University, Chile

\section{ARTICLE INFO}

Received: 幽 July 02, 2020

Published: 幽 July 17, 2020

Citation: Marcos Baabor A, Hernán Delso P, Bayron Valenzuela C. The Translaminar Microsurgical Approach to Lumbar Disc Herniation with Foraminal Stenosis: A Modification to the Traditional Surgery Technique. Biomed J Sci \& Tech Res 29(1)2020. BJSTR. MS.ID.004731.
ABSTRACT

At the Clinical Hospital of the University of Chile, we have used the translaminar approach to access the Hidden Zone (HZ) and the Foraminal Area. To achieve this objective, the technique described by Di Lorenzo and modified posteriorly by Bernucci and Soldner was used in two clinical cases. The first case shows the clinical and radiological response to surgical treatment of an extruded foraminal ascending Lumbar Disc Herniation (LDH) through a translaminar window. The second case shows a Lumbar disc herniation to the HZ with a concomitant foraminal stenosis, so a modified translaminar approach is proposed to extract the extruded disc fragment and to explore the impinged nerve root through the translaminar window. Both cases with a 6 months follow-up with good clinical and radiological outcomes. The surgical technique and its modifications are described, discussing its scope and usefulness. This minimally invasive technique allows, in most cases, an early hospital discharge and early clinical outcomes. The medium-term controls (6months) show good clinicalradiological evolution, without signs of lumbar instability of the operated segment.

\section{Introduction}

Disc Herniation corresponds to the protrusion of the central gelatinous material of an intervertebral disc through a fissure in the annulus. It may break off completely with disc material coming out to the epidural space, or it may remain partially intact and lead only to disc protrusion. This process can be associated with low back pain due to the increase in sensitivity of the nerve root derived from inflammation and/or compression of the nerve root and its ganglion, associated or not with paresthesia or paresis of the lower extremities [1]. Between the different patterns of LDH, most compress the descending nerve root at the level of the intervertebral space. However, there are some less frequently presentations, that compress the exiting root at the level of the intervertebral foramen, implying a greater difficulty in its surgical approach [2]. These presentations, depending on the location in relation to the position of the joint pedicles, can be classified as Extraforaminal or Far Lateral (External to the margin that connects both adjacent pedicles), Foraminal (Between the Medial and Lateral border of both pedicles) or Hidden Zone (HZ) Hernias, located towards medial of the medial margin that connects both pedicles, described by Macnab [3]. In the search for a better approach for ascended LDH located at the level of the foramen with root compression, [4] describes a surgical access through a fenestration (10x 5mm) in the bone at the level of the lamina with its longitudinal axis greater at the level of the pars interarticularis located medial and slightly off-centered to the lateral isthmic notch, leaving a margin of 3-mm rim of bone, in the lateral aspect of the pars to prevent iatrogenic fractures [4]. This would permit the extraction of the LDH fragment over a traditional interlaminar access. In this way, the extruded fragment in the $\mathrm{HZ}$ is reached, avoiding the need to perform a wide and high laminectomy (with involvement of the Isthmus and pars interarticularis) which would precipitate future iatrogenic spondylolysis with instability of the lumbar segment, with its clinical and surgical consequences. 
Subsequently, several studies have demonstrated the effectiveness of this approach, making modifications to the original technique proposed by Di Lorenzo et al. In their work, Soldner et al. [5] describe Di Lorenzo's technique as "fenestration in the Pars Interarticularis", theirs being described as "Translaminar fenestration", consisting in a laminar fenestration located craniomedial to the articular facet. The location of the fenestration depends on the corresponding location of the extruded LDH fragment. Bernucci et al. [6] describes a technique like the one described by Soldner, but they add a traditional interlaminar approach, leaving the lower edge of the upper sheet intact allowing the exploration of the disc space from which the extruded fragment originated. In cases where the extraction of large fragments through the opening cannot be performed, they could perform the extraction through the interlaminar approach. Among the main criticisms made to the surgical approach described by Di Lorenzo, was the impossibility of being able to perform additional removal of disc tissue in the disc space. Likewise, other commentaries pointed to the possibility that adding a laminar fenestration and thinning the cortical bone layer of the adjacent pars interarticularis could, even if an iatrogenic fracture was not performed at the time of surgery, eventually develop a fracture in a postoperative manner associated with minor trauma [4]. However, multiple studies conducted posteriorly with radiological follow-up at 6 and 12 months showed no laminar o Pars interarticularis fractures or radiological signs of spinal instability [5-7].

The transforaminal approach implies a thorough knowledge of the spinal anatomy and specially, the anatomy corresponding to the $\mathrm{HZ}$ and the Intervertebral Foramen. The foramen anatomy corresponds to the shape of a square: 1 . The roof formed by the lower edge of the upper pedicle; a posterior wall corresponding, from cephalic to caudal, Pars Interarticularis, free edge with ligamentum flavum, facet joint and upper articular facet of the lower vertebra; the floor formed by the upper edge of the lower pedicle; an anterior wall formed by the intervertebral disc on its posterior edge and the posterior wall of the upper vertebra (Figure 1) [8]. The Hidden Zone corresponds to the area located towards the middle of the edge of the pars interarticularis, located below the Vertebral Lamina, described by Macnab [3]. Among the studies carried out on the foraminal area, Silverstein et al. [9] perform a study to determine the location of the lumbar dorsal root ganglion. This study analyzed 16 embalmed corpses using Thoraco-Lumbar MRI and subsequent dissection, to investigate the dimensions of the lumbar intervertebral foramen and the localization and wide of the Dorsal Root Ganglion. An anatomical division of the foraminal zone is made into 5 regions from medial to lateral, described in Figure 2. In this study, it was determined that the center of the dorsal root ganglion is preferably located bilaterally in the medial zone of the foramen (Zone 3) between L1 to L4 and in the lateral zone to the foramen (Zone 4) at L5. [9]. This is how the approach through translaminar fenestration allows to locate mostly in this area, the dorsal root ganglion and the axilla of the exiting root at the same level. In addition, if we reject the root towards the midline, we expose the shoulder of the nerve root and the lower edge of the pedicle.
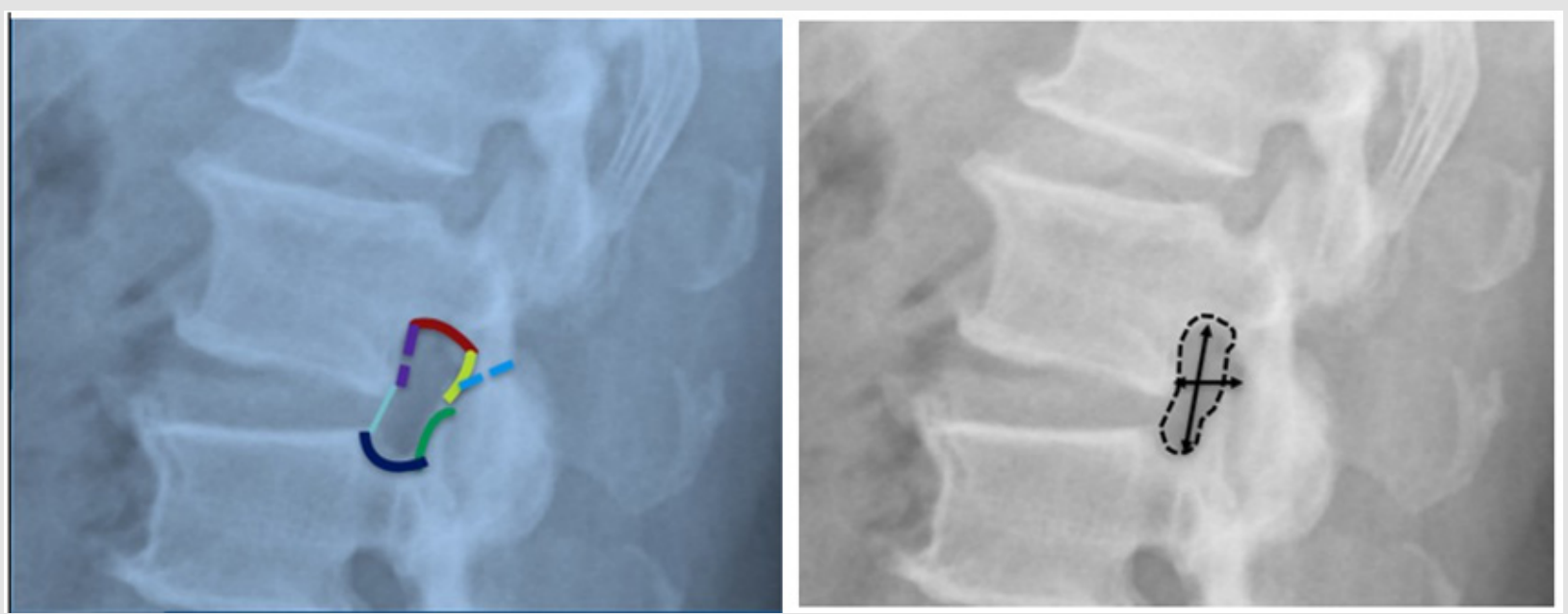

Figure 1: Limits of the Intervertebral Foramen: Roof formed by the lower edge of the upper pedicle, Posterior wall corresponding from cephalic to caudal, by Pars Interarticularis, free edge with ligamentum flavum, facet joint and upper articular facet; Floor formed by the upper edge of the lower pedicle; Anterior wall formed by the intervertebral disc on its rear edge and the rear wall of the upper vertebra. On the right you can see the average diameters: Longitudinal diameter Average distance 17-22mm, Anterior to posterior diameter 8-10mm. Intervertebral Foramen Area 40-160 $\mathrm{mm}^{2}$ [10]. 


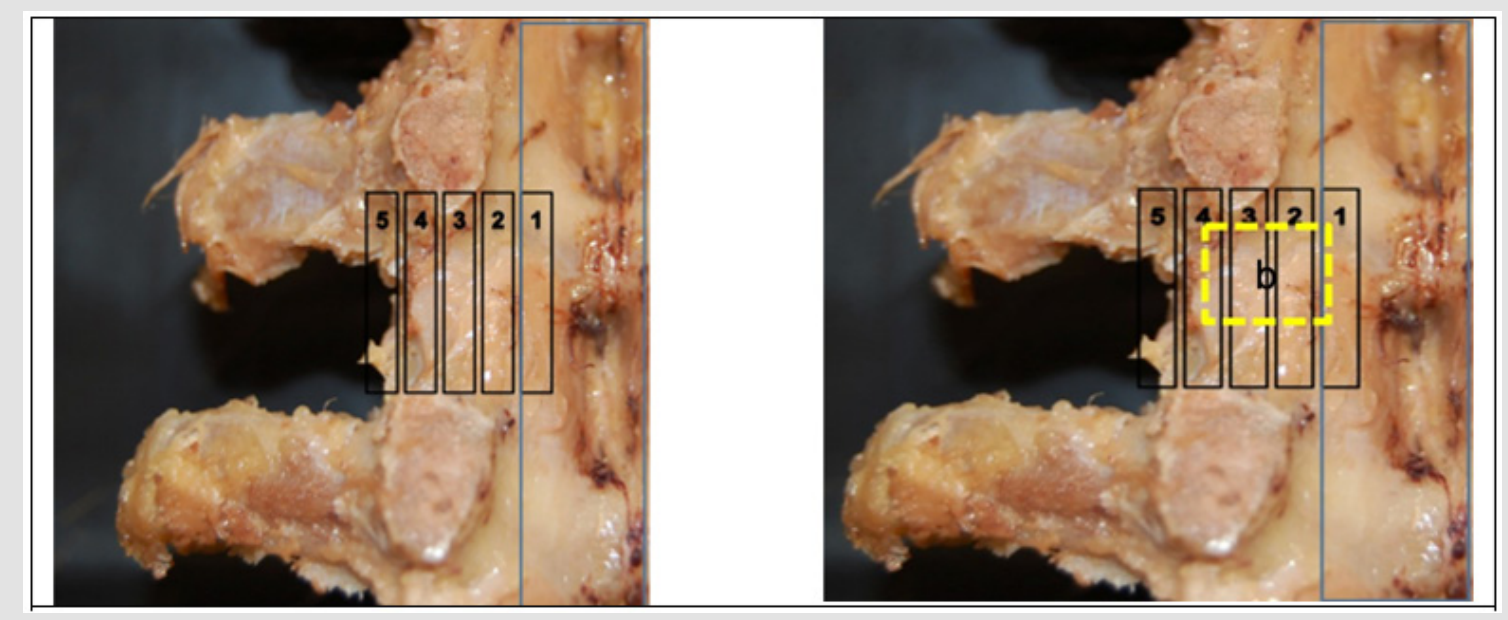

Figure 2: Anatomical Division of the Foraminal space: Zone 1 and 2 Intra-Spinal, Zone 3 Medial Neuroforamen, Zone 4 Lateral Neuroforamen and Zone 5 Extraforaminal Space. The image on the right shows the location of the Transforaminal approach proposed by our team. Images taken from the study by Silverstein et al. [11]

\section{Materials and Methods}

At the Clinical Hospital of the University of Chile, we recently began to adopt the surgical technique to address Lumbar Foraminal Stenosis, Extruded LDH with ascending compression against the lower edge of the pedicle and Ascending Recurrent LDH, in stable lumbar columns. We present two clinical cases addressed in our hospital. The first case shows the adoption of the classical technique described by Bernucci [6]. In the second clinical case, we describe a modification of the surgical technique, performed in a patient with foraminal stenosis concomitant with the ascended LDH, with which we seek to develop an approach to the exiting nerve root in its entirety.

\section{Surgical Technique}

Patient under general anesthesia in supine position, with antibiotic prophylaxis. Antisepsis local with chlorhexidine. With Spinal needle, the upper vertebra is marked at the level of the spinous process, and position is visualized with Fluoroscopy in Lateral mode to determine the lumbar level to be operated on. A 2 to $3 \mathrm{~cm}$ midline incision is made, centered in relation to the marking. Subcutaneous tissue is sharply divided. Opening of the muscle fascia in a longitudinal fashion parallel to the skin incision. Paraspinal muscle are elevated digitally, and then subperiosteal fashion with sharp subperiosteal elevator on the ipsilateral side to the herniated disc only. Once the upper vertebra hemilamina has been exposed, a new fluoroscopic evaluation is performed to check the corresponding disc space. Muscle retraction is performed with Micro Taylor Spinal Retractor $(1,2 \mathrm{~cm})$ and the Microscope is brought into position. With a high-speed drill, a fenestration of $6 \times 6 \mathrm{~mm}$ is made in the Lamina, where it is expected to cross 3 bone layers: an external cortical, then spongy tissue and then internal cortical. The location is towards the caudal and medial edge of the pars interarticularis. According to the lumbar level to perform the translaminar fenestration, the distance in millimeters from the edge of the pars interarticularis is left as described by Reulen et al. [10]. The upper edge of the ligamentum flavum is removed with Kerrison rongeur, to enter the epidural space. The space is then explored with a dissector and a blunt nerve hook. In this way, the Axilla of the exiting nerve root and sometimes the descending root are visualized. The extruded fragment is located with displacement and compression of the compromised root. Using biopsy forceps and fine dissectors, the extruded fragment is extracted and raised to the level of the foramen. A standard wound closure is then performed. In the second case, a modification of the standard technique described above is performed. In this case, the patient presented a foraminal stenosis. It was decided then, to enlarge the foramen by opening the lateral edge of the translaminar fenestration, which allows the vision of the protruding root to be enlarged along its entire length, allowing it to be released in all its magnitude.

\section{Case 1}

A 76-year-old male patient begin with sudden paresia in his left lower extremity associated with left thigh pain with inability to walk. On neurological examination, neurogenic claudication is evident, associated with abolished Patellar Reflex, and numbness in L4 and L5 territory. MRI images of the lumbar spine show extruded and ascended hernia at the left L4-L5 foramen (Figure 3). A left L4 translaminar approach with extruded fragment resection is planned. Subsequent control is performed with CT of the lumbar spine and with 3D bone reconstruction (Figures 4 \& 5). Ambulatory control with functional X-Ray does not show spine instability in 6 months control. Clinical outcome evaluated with VAS show an improvement in immediate pain (from $7 / 10$ to $1 / 10$ ) and L4 paresis at 6months, only the Patellar reflex shows a partial improvement, with slight asymmetry persisting. 


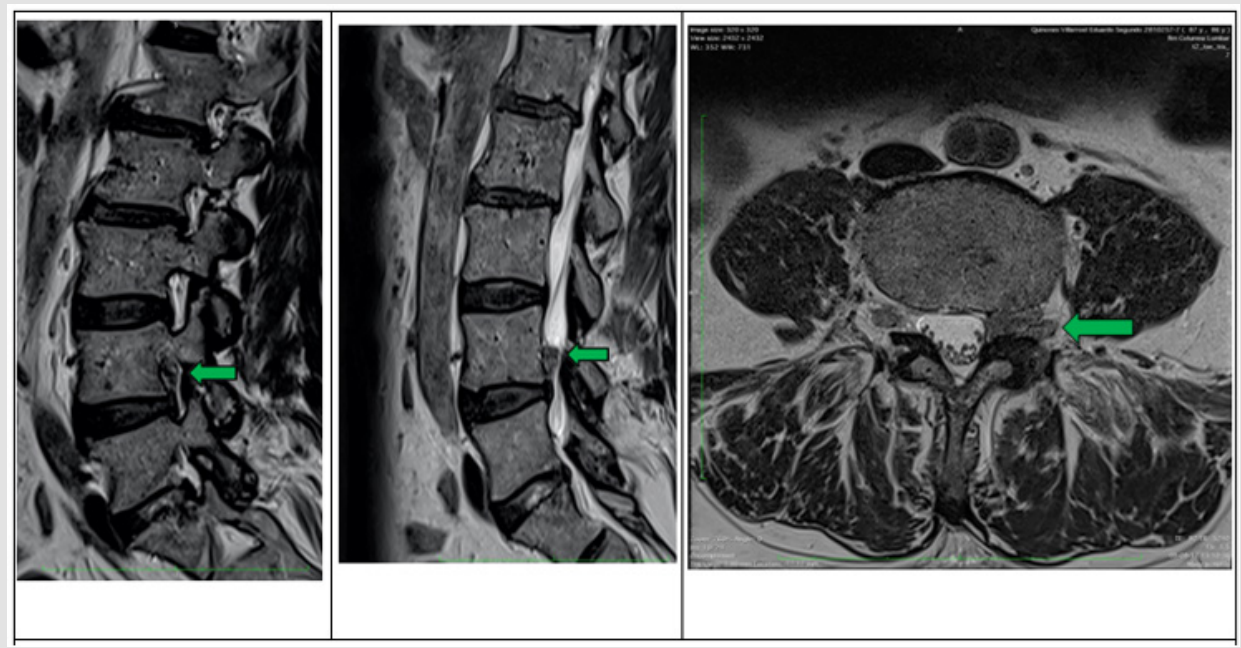

Figure 3: Lumbar MRI in T1 Sequence. The Arrows indicate the extruded fragment of ascended Disk L4 located at Foraminal level.

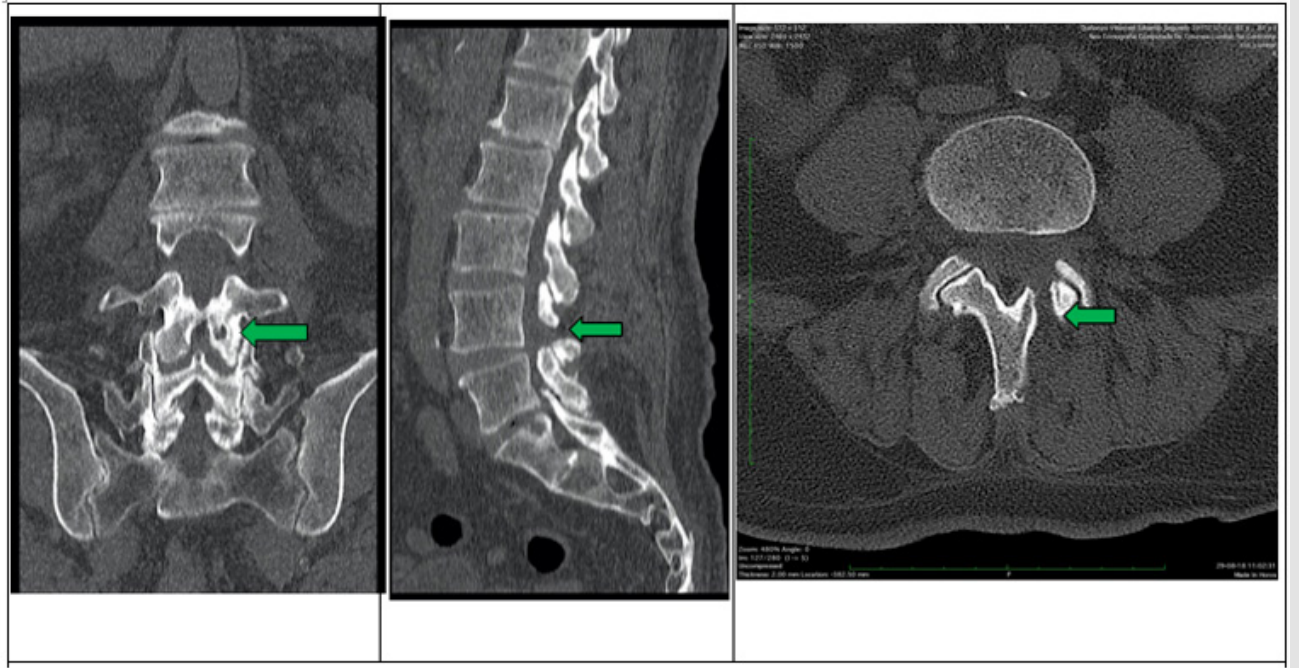

Figure 4: Lumbar MRI in T1 Sequence. The arrows indicate where the L4 Translaminar Fenestration was performed.

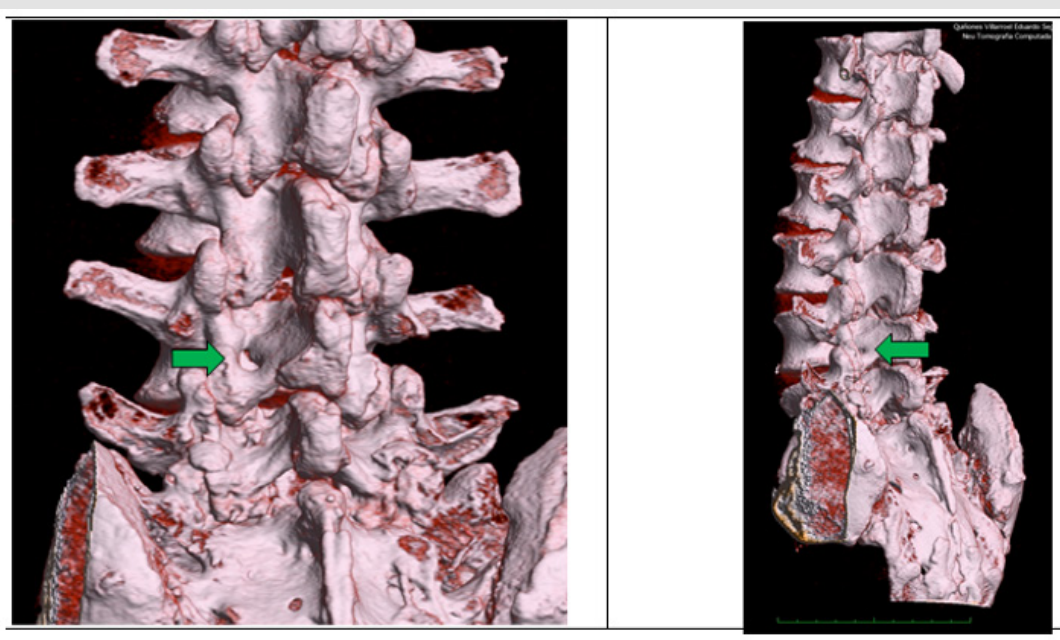

Figure 5: 3D Bone Reconstruction from Axial Computed Tomography. The arrows mark the translaminar site where the fenestration was performed. 


\section{Case 2}

A Male patient of 57 years of age, evolves with pain in lumbar region and right side, with extension to right gluteus and popliteal zone, adding paresthesia with neurogenic claudication, paresia at level of hip and thigh. Duration of clinical symptoms of 6 months. MRI of lumbar spine is performed, showing Foraminal Stenosis associated with Right L1-L2 LDH (Figure 6). A Translaminar Approach was performed (L1 right) with a resection of LDH extruded fragment and enlargement of the L1 intervertebral Foramen. Patient refers functional recovery during the immediate postoperative period with improvement of pain and no sensory disturbances and starts walking with Kinesiotherapy within the same day. Posteriorly, it was evaluated with postoperative MRI and CT of the lumbar spine with bone reconstruction (Figures 6-9). In a 6-month postoperative control, no radiological elements of spinal instability were seen in a dynamic radiographic study. Neurological evaluation at 6 months is normal.

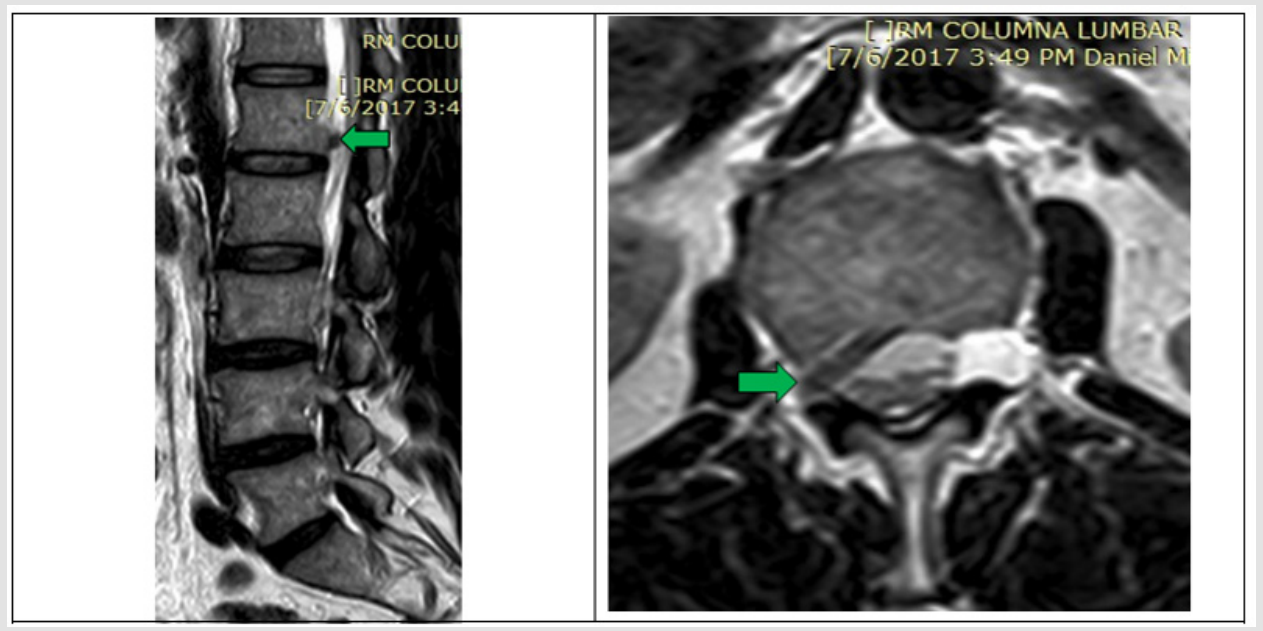

Figure 6: Sagittal and Axial Section of MRI Sequence T1 of the lumbar spine. L1-L2 extruded fragment located at foraminal level with L1-L2 Root compression can be displayed. Arrow marks in both cases Extruded Fragment.

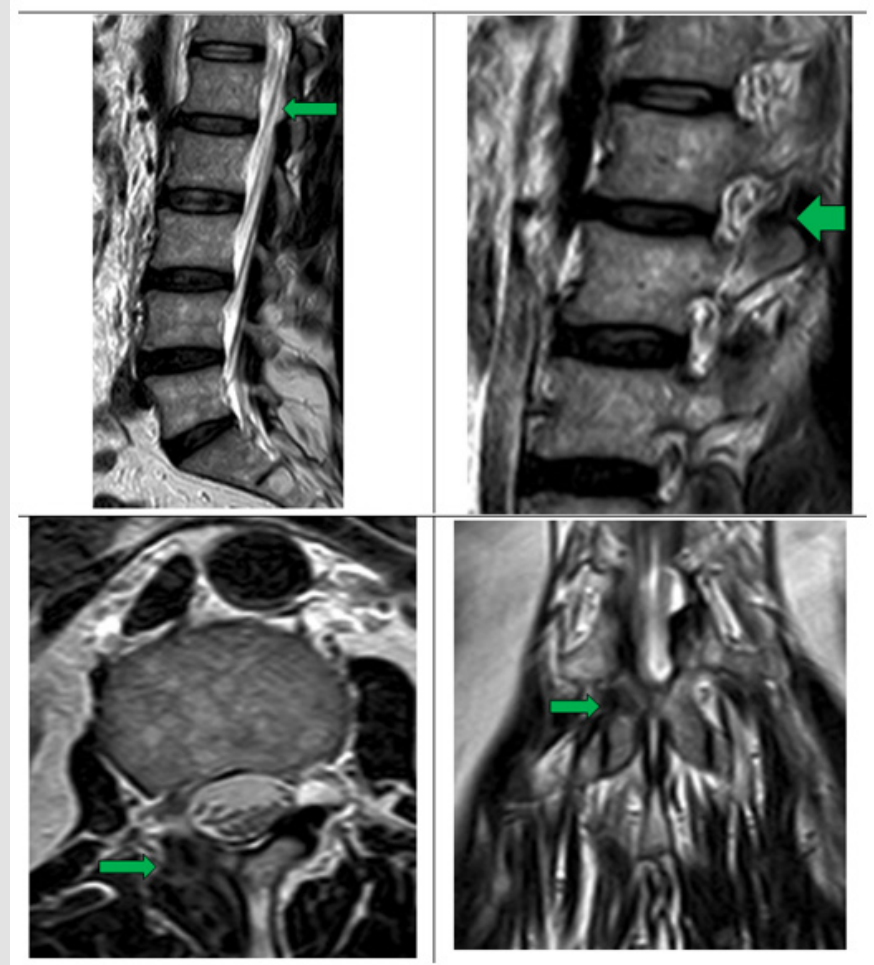

Figure 7: Sagittal and Axial section of MRI Sequence T1 of lumbar spine in postoperative evaluation. Currently, no extruded fragment is observed at the L1-L2 level. Arrows mark the place of the translaminar fenestration and the enlargement of the intervertebral foramen. 


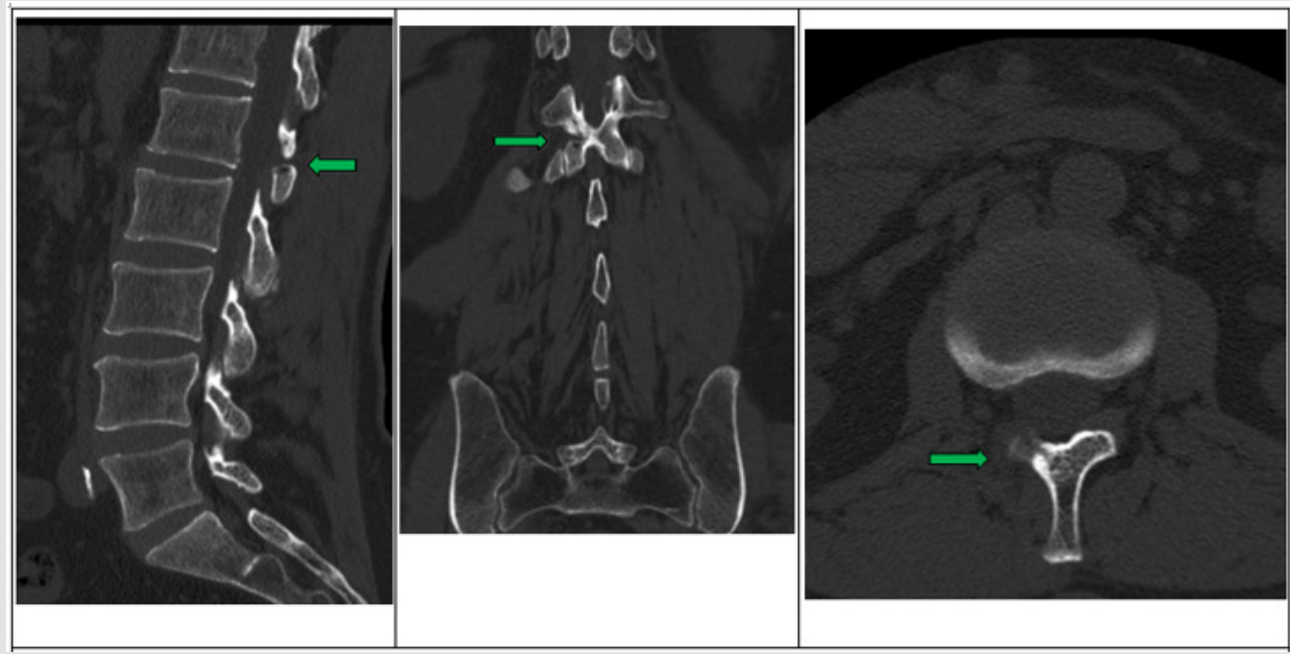

Figure 8: Computed tomography with Bone window. Arrows show enlargement of the foramen and translaminar fenestration of L1.

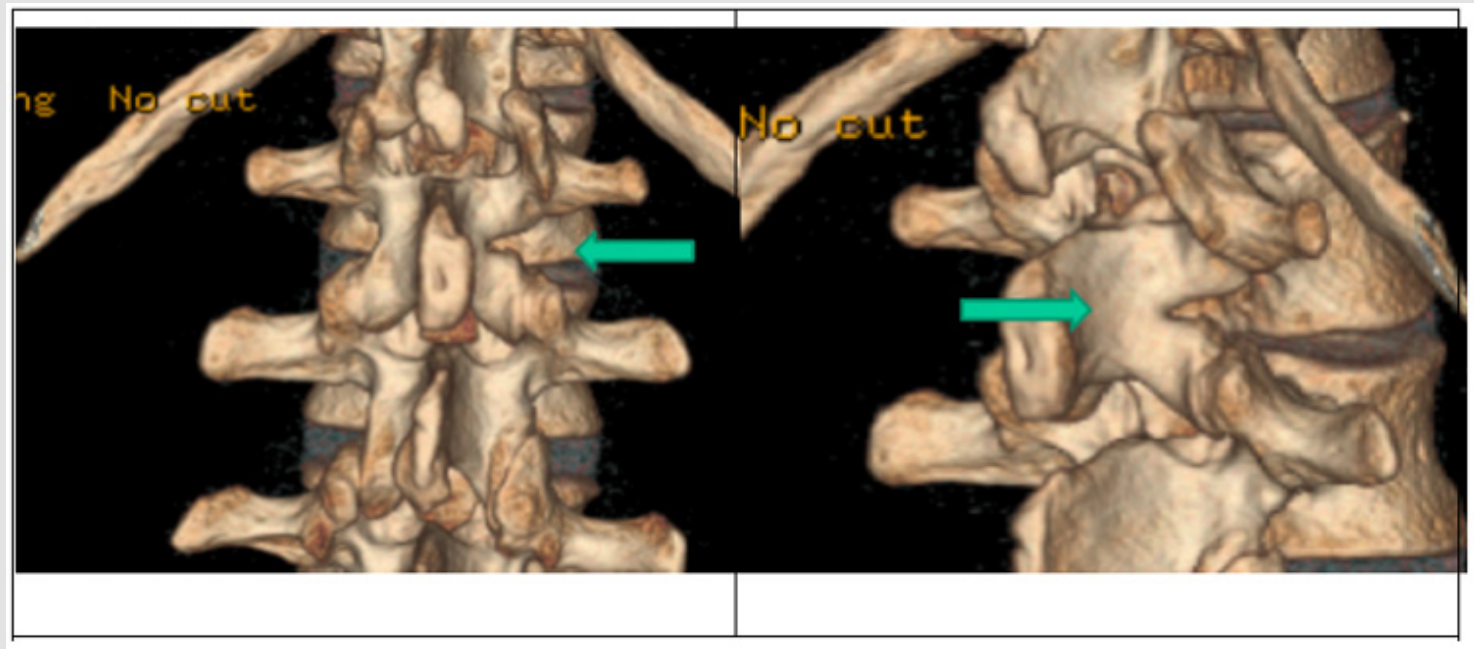

Figure 9:3D Bone Reconstruction from Lumbar Spine CT The arrows indicate the area where the Foramen and the Translaminar approach are to be extended.

\section{Discussion}

In the first case, we performed a translaminar approach for an LDH with an extruded component ascended with compression of the L4 root, located near the HZ. In this case, the translaminar opening was studied with a pre-operative workup with MRI and CT to identify the best location for the fenestration. Previously described by other authors [5-8], the preoperative study is crucial to locate the relationship of the extruded fragment to perform the translaminar approach. Likewise, the anatomical study of the HZ proposed by Macnab [3] and applied in subsequent investigations in anatomical-radiological relation [11] allows for the comprehensive management of the nerve root compression at the HZ level. In the second case, when the laminar window is opened and the epidural space explored, the first thing that can be examined under the microscope is the exiting root in all its magnitude, axilla and shoulder. Posteriorly, exploring with a blunt nerve hook, extruded fragments are located and extracted. If we incline the microscope caudal, it can observe the fissured disc and even if necessary, local exploration can be achieved, which would even limit the need for the complementary interlaminar approach proposed by Bernucci in his modification to the Di Lorenzo technique [6]. One of the main criticisms made to the Transforaminal approach initially proposed by Di Lorenzo, consists of the weakening of the area right next to the pars interarticularis, with increased potential risk of fracture and subsequent spondylolysis. However, studies performed posteriorly with a transforaminal approach [5-7] demonstrated, in the long term at 6 and 12 months, with clinical and imaging followup the absence of fracture and spinal lumbar instability, with a good clinical outcome in the Macnab and VAS criteria in about 75- $80 \%$ of the cases. In these two cases presented, a good clinical recovery was evidenced in the long term, the imaging follow-up with functional X-ray at 6 months showed no lumbar spinal instability or signs of 
joint fracture, and improvement in the clinical outcome with similar results like the previously reports in the Macnab criteria.

In case number 2, we modified the traditional translaminar surgical technique with the opening of the laminar window towards the lateral edge, (corresponding to Zone 4, or lateral foraminal), which allows us to extend the surgical field to visualize the nerve root in its trajectory from the area of the axilla to its emergence in the intervertebral foramen, including the extraforaminal area. This allows in cases of foraminal stenosis, to release the root all along its course. This approach would be indicated in foraminal stenosis with exiting root involvement. This technique shows great advantages since it aims to manage the compressed exiting root, being minimally invasive with respect to the joints, muscles and ligaments, thus preserving stability. In addition, the root is approached from proximal to distal, reaching the sensory ganglion from the midline, which makes radiculitis pain less frequent than if it is approached from distal to proximal. In the future, the question remains as to whether the application of this modification would have a greater long-term impact (greater than 6 months) on lumbar spinal instability and spondylolysis, to evaluate the effectiveness of foraminal stenosis management in a broader case report, and to define the criteria for patient selection, in order to enhance it in the future.

\section{Conclusion}

This is a minimally invasive microsurgical technique, very useful in the approach of the Foraminal zone with the aim of decompressing it either by LDH, fibrosis or stenosis. With a good knowledge of the anatomy of the intervertebral foramen and the $\mathrm{HZ}$ and proper training, it is easy to perform. The clinical and anatomical results for the 2 cases were optimal, allowing an early hospital discharge, start walking within the same day of surgery, and with medium-term controls at 6 months with good clinical- radiological evolution, without advancing with spinal instability. The modified technique for exiting nerve root Foraminotomy, with enlargement of the intervertebral foramen, needs to increase its casuistry and follow-up to obtain definitive results, and continue its long-term analysis to determine possible future complications.

\section{References}

1. Guía Clínica (2013-2014) Hernia de Núcleo Pulposo Lumbar. SANTIAGO: Minsal.

2. Diaz-Ledezma C, Urrutia J, Romeo J, Chelen A, González-Wilhelm L, et al. (2009) Factors associated with variability in length of sick leave because of acute low back pain in Chile. Spine J 9(12): 1010-1015.

3. Macnab I (1971) Negative disc exploration. J Bone Joint Surg Am 53(5): 891-903.

4. PH Young (1998) Pars interarticularis fenestration in the treatment of foraminal lumbar disc herniation: a further surgical approach. Neurosurgery 43(2): 87-90.

5. Soldner F, Hoelper BM, Wallenfang T, R Behr (2002) The translaminar approach to canalicular and cranio- dorsolateral lumbar disc herniations. Acta Neurochir (Wien) 144(4): 315-320.

6. Bernucci C, Giovanelli M (2007) Translaminar micro- surgical approach for lumbar herniated nucleus pulposus (HNP) in the hidden zone: clinical and radiologic results in a series of 24 patients. Spine (Phila Pa 1976) 32(2): 281-284.

7. Seong Son, Sang Gu Lee, Woo Kyung Kim, Yong Ahn (2018) Advantages of a Microsurgical Translaminar Approach (Keyhole Laminotomy) for Upper Lumbar Disc Herniation Neurosurg 119: e16-e22.

8. Russel V. Gilchrist, Curtis W Slipman, Sarjoo M Bhagia (2002) Anatomy of the Intervertebral Foramen An Anatomical Review. Pain Physician Oct 5(4): 372-378.

9. Reulen HJ, Pfaundler S, Ebeling U (1987) The lateral microsurgical approach to the extracanalicular lumbar disc herniation. I: A technical note. Acta Neurochir (Wien) 84(1-2): 64-67.

10. Papavero L, Kothe R (2013) The translaminar approach for cranially extruded lumbar disc herniations. Oper Orthop Traumatol 25(1):6-15.

11. Michael P Silverstein, Lynn JR, Edward CB, Nicolas T, Sandra G, et al. (2015) Lumbar Dorsal Root Ganglia Location: An Anatomic and MRI Assessment. Michael P Silverstein, MD et Al. Int J Spine Surg 9: 1-10.
ISSN: 2574-1241

DOI: $10.26717 / B J S T R .2020 .29 .004731$

Marcos Baabor A. Biomed J Sci \& Tech Res

CC (i) This work is licensed under Creative

Submission Link: https://biomedres.us/submit-manuscript.php

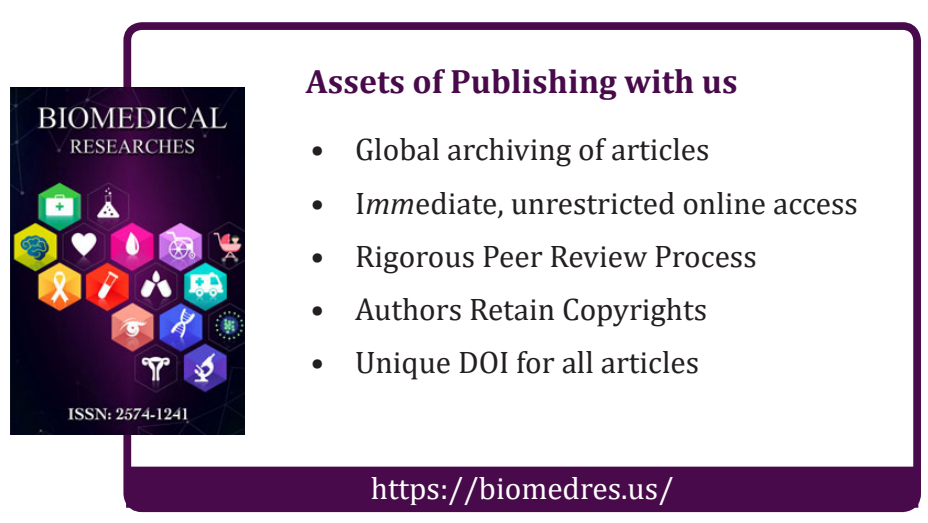

